\title{
Mechanical design of the RPC-based ToF wall (iTOF) for the R3B experiment at FAIR
}

\author{
E. Casarejos*, J.A. Vilán, P. Izquierdo, M. López-Lago, A. Segade \\ University of Vigo, E-36310 Spain \\ E-mail: e.casarejos@uvigo.es
}

Y. Ayyad, J. Benlliure, I. Durán, C. Paradela, J.R. Pereira

University of Santiago de Compostela, E-15782 Spain

\begin{abstract}
The design of iTOF, a strip RPC based time-of-flight detector dedicated to measure relativistic heavy ions, is presented here. The proposed design includes innovative solutions, as the selfsupported RPC concept, which solves the specific needs of working with heavy ions. The proposal is based on the results of the intense previous $R \& D$ program undertaken by our group to build and test prototypes with ion beams. The project is developed within the frame of the R3B-FAIR activities.
\end{abstract}

XI Workshop on Resistive Plate Chambers and Related Detectors

5-10 February, 2012

Laboratori Nazionali di Frascati dell'INFN - Frascati (Rome) - Italy

\footnotetext{
* Speaker.
} 


\section{Introduction}

Resistive plate chambers (RPCs) with submilimetric gas gaps and high electric fields (100 $\mathrm{kV} / \mathrm{cm}$ ) make it possible to achieve low time-resolution values and cover large surfaces with costeffective granularity. HEP experiments worldwide have built and commissioned timing RPC setups with time resolutions better than 100 ps (sigma value): ALICE [1] at CERN, FOPI [2] and HADES [3] at GSI, STAR [4] at BNL. All of these detectors were developed to measure minimum ionizing particles (MIPs). The main challenge, beyond resolution, was to overcome the limited efficiency of a single gas gap [5]. That was possible with the use of RPC configurations of multiple gaps $[6,7]$. After years of $R \& D$ and daily working experience accumulated, the conditions to run these RPCs are well established in what concern the field value (close to $100 \mathrm{kV} / \mathrm{cm}$ ) and the right gas mixtures, most of them based on the combination of tetrafluoroethane $\left(\mathrm{C}_{2} \mathrm{H}_{2} \mathrm{~F}_{4}\right)$ and sulphur hexafluoride ( $\mathrm{SF}_{6}$, below $10 \%$ ), and sometimes the addition of isobutane (iso- $\mathrm{C}_{4} \mathrm{H}_{1} 0$, below $5 \%$ ).

The possible application of these detectors to other scenarios (increasing rate capabilities, tracking, ...) has called the attention of R\&D efforts in recent years. Our group proposed the use of RPCs as a feasible and competitive solution for time-of-flight (ToF) detectors dedicated to measure relativistic ions, both light and heavy [8]. The application would be suited to the case of the R3B experiment at the large FAIR facility [9]. The R3B experimental program includes the study of fission reactions with complete kinematics. This kind of experiments require $100 \%$ efficient detectors with time resolutions good enough to identify nuclei with masses up to 150 atomic number units. For moderate ToF distances, about 15 meters, it requires time resolution values below 70 ps (FWHM).

By the time we started our activities there was not available information about the RPCs detecting heavy ions, the first appearing in Ref. [10]. Our R\&D project for implementing this technology in this domain covered the design of the RPC (size, geometry, granularity, suited materials, number of gaps, field distribution, ...), as well as the (very-) front-end electronics (vFEE) to couple to the detector. To define all the pieces of the puzzle, we developed a wide program of prototype construction and tests with beams of ions at the Helmholtzzentrum für Schwerionenforschung GmbH Darmstadt, Germany (GSI). The results were modeling the conceptual ideas of a suited design for the R3B conditions. After years of R\&D we propose a design with proven results about the performance of the RPCs with heavy ions based on the results of Refs. [11, 12, 13, 14]. This detector is called iTOF.

\section{2. iTOF requirements}

The studies for which iTOF is developed cover the production of heavy and medium mass nuclei after fragmentation (peripheral) reactions of heavy nuclei. In these conditions one has few relativistic residues after fission and/or particle-emission processes, to be identified. In R3B the residues are analyzed with a dipole magnet. In the general case, the identification methodology is based on the combination of charge-sensitive detectors to measure energy losses and identify the ion charge, tracking detectors to define the magnetic rigidity, and ToF detectors to measure the velocity. 
We used a GEANT4 based simulation to transport the ions after the reaction vertex, through the large aperture dipole magnet, till the detection planes. In the case of heavy ions the right description of both energy and angular straggling, corresponding to the elastic processes during the transport through the layers of matter of the setup, is critical. A study case is that of fission at the lower energies of interest in R3B, like the fission of ${ }^{238} \mathrm{U}$ at $400 \mathrm{MeV} / \mathrm{u}$. In order to produce reliable results one needs to use an event generator based on state-of-the-art models able to provide the right description of the reaction mechanisms of interest [15]. The angular aperture induced by fission (up to $50 \mathrm{mrad}$ ), and the following transport and dispersion in the dipole magnet, results in a transmission surface of $1 \mathrm{~m}$ in the vertical direction, and $2 \mathrm{~m}$ in the horizontal/dispersion direction, considering a feasible flight-path of $15 \mathrm{~m}$.

The fission residues with mass numbers up to values of 150 , require a time-resolution of $70 \mathrm{ps}$ (FWHM) to fully separate two isotopic species. Considering that the final time resolution includes both the value of the start timing detector, and the RPCs, we proposed to 'slice' the detector into several planes of independent measurement. Therefore for $\mathrm{N}$ planes of detection we gain a rough factor $\sqrt{N}$ in the time resolution of the the combined measurements. In that way we can make compatible the resolutions demanded in the setup, and the feasible RPC resolution values with the actual technologies.

Another study case is that of particle-emission residues of a heavy ion, as it is the fragmentation of a ${ }^{208} \mathrm{~Pb}$ beam. The mass range, above 200 mass units, demands a time-resolution of some 50 ps (FWHM) for the same flight-path, but the angular aperture is much smaller in this case (below $5 \mathrm{mrad}$ ). To face this case with the same RPC detectors, one can either increase the flight-path to reduce the time-resolution requirement to RPC feasible values; or to increase the number of detection planes, to achieve a good enough time resolution to solve this case. Therefore we wanted to include in our design the possibility to change the number of measuring planes, as well as changing the surface and geometry of the detector by building it in a full modular configuration.

To accurately define the velocity the impact position has to be determined to correct the length of the flight-path, therefore correcting the angular straggling after a flight of 15 meters. That is possible if the detector has enough granularity, and/or measuring the position with a resolution value below $10 \mathrm{~mm}$. Another issue is that of the multi-hit capabilities. Fission reactions include medium-mass residues accompanied mostly by protons (neutrons will not be deflected by the R3B dipole, and will arrive to specific detectors). Particle-emission processes of ions produce heavymass residues together with protons and alphas, and in minor quantity other light charged particles. Other processes as multi-fragmentation and light-charged-particle emission, increase the multiplicity. The granularity of the detector should resolve the multi-hit requirements.

\section{3. iToF modules: structural concepts}

To cope with the above mentioned requirements we proposed to design iTOF as a collection of identical modules, with an active area of $100 \mathrm{~cm}$ length and $35 \mathrm{~cm}$ width, based on multi-strip RPCs layers. The RPC are made with glass plates (all details will be given in the next section). Due to the large energy loss of the ions through any layer of matter, and the consequences of degrading the detection if too much matter was used in the detector itself ${ }^{1}$, we developed the concept of

\footnotetext{
${ }^{1}$ The amount of glass that ${ }^{208} \mathrm{~Pb}$ at $400 \mathrm{MeV} / \mathrm{u}$ can pass through before stopping is just below $15 \mathrm{~mm}$.
} 

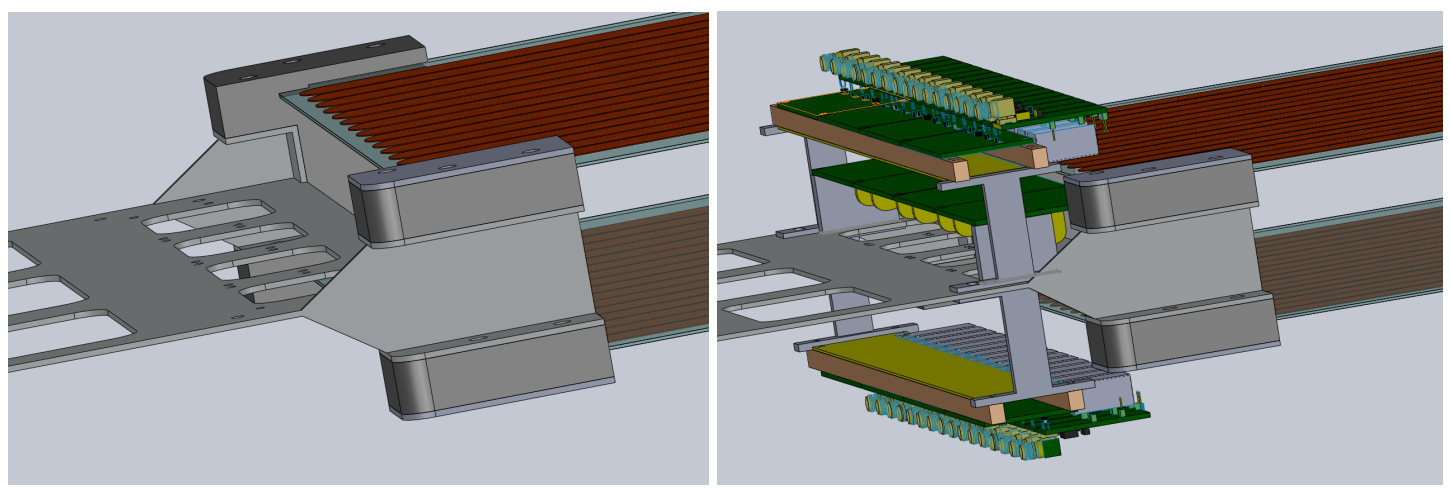

Figure 1: Design of the iTOF module. Left panel: Two self-supported one-gap RPCs are set together by using custom polyurethane pieces which hold the two cores at each corner. A stainless-steel plate with arms tight all the pieces together, fixing the distance between the RPCs. Right panel: View of a side of the module. The HV distribution boards are located at the same level of the glass-plates to minimize the noise due to the wiring. The vFEE boards are made for individual channels, and are located just above the strip edges. A second type of board, placed just below the vFEE level, regulates the power supply.

'self-supported RPC' with a reduced amount of material in the active region. The thickness of the glass plates is the minimum that allows for a robust construction with no need of any other layer of structural material to hold the RPC and interfering with the active region.

To produce functional and robust structures we proposed to use the 'sandwich' concept to build the modules. Our design mounts two (self-supported) RPC layers in the same module, sharing the structural frame. With this configuration we obtain a compact module, with two autonomous measuring planes. The module made in this way in much more robust, as we have calculated with structural evaluation codes (finite elements models). Additionally being the two RPC layers arranged to have their strips shifted by a half-pitch, the dead space is minimized, as well as the multi-hit missing events, when combining the information of the two layers. Another asset of this configuration is the reduction of noise to signal ratio by setting the ground planes at the external surfaces of the module. This also make a safer operation module avoiding extra voltage protection layers around the module.

The two self-supported RPCs of the module are hold with custom-made polyurethane pieces, shore 40 hardness, which clamp the two layers at each corner, see figure 1, left panel. A stainlesssteel plate with arms tight all the pieces together, fixing the distance between the RPCs $(6 \mathrm{~cm})$, see figure 1, left panel. To make an operating module we also need to accommodate the boards of vFEE and their power regulators, and the HV filters and splitters. On both sides of the plate, the boards are placed using ad-hoc spacers, to have both the right clearances and the minimum signal path from the strip, see figure 1, right panel. Each RPC is biased at one side. The HV distribution boards are located at the same level of the glass-plates to minimize the noise due to the wiring. The voltage is fed through a load resistor to one of the strips while the opposite strip (outer surface of the module) is grounded. The guard strips are also grounded. The signal pick-up points are at both sides of the strips. The vFEE boards are made for individual channels, and are set just above the strips. The signal is feed to the vFEE with a decoupling capacitor from one strip (biased), and directly wired from the paired (grounded) strip. An SMA-type connector brings the signal to the 

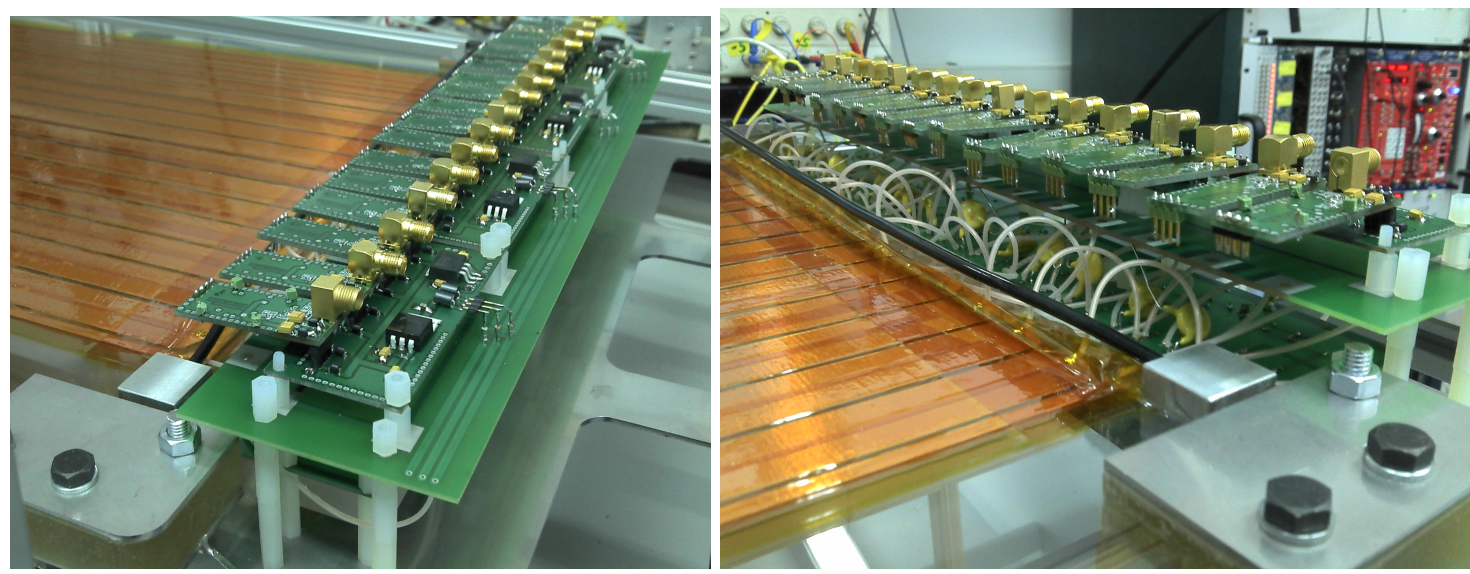

Figure 2: Construction of a module: two views of a side of a module prototype. We can see the polyurethane piece holding the self-supported one-gap RPCs, tightened by an external metal plate. The gas injection piece is placed next to glass edge. The vFEE boards for individual channels are located just above the strip edges, in the top level. The boards which regulates the power supply are just below. The HV distribution boards are located at the same level of the glass-plates to minimize the noise due to the wiring, clearly visible in the right panel.

next front-end stage for digitalization. A second type of board, placed just below the vFEE level, distributes the low-voltage power supply to the vFEE, grouped by 4 channels. In figure 2 we show two views of a prototype built to probe the design concepts. After the construction, it will be fully operative and ready for characterization.

The iTOF modules built in this way are single measuring units than can be safely handled and positioned in convenient frames to setup the detector to cover different surfaces, geometries and number of planes of measurement. A collection of 6 modules, with one strip of overlap between two of them, and a zig-zag positioning, covers a surface of $2 \mathrm{~m} \times 1 \mathrm{~m}$, with the strips in vertical direction, see figure 3. A second plane of modules, either with vertical or horizontal strip alignment, would make a setup with four planes of time measurement. This modularity make it possible to adapt the number of planes and surface geometry to each reaction case according to its particular kinematics.

\section{The self-supported RPCs of iTOF}

With the requirements mentioned above we proposed to design iTOF as a collection of identical modules, with an active area of $100 \mathrm{~cm}$ length and $35 \mathrm{~cm}$ width, The RPCs are based on a multi-strip design with 16 strips and a single gas-gap, made with two plates of commercial sodalime glass, $1 \mathrm{~mm}$ thick. Nylon fishing line is used as spacer for creating the gas gap of $0.3 \mathrm{~mm}$ between plates. The strips, $100 \mathrm{~cm}$ long, are made of self-adhesive copper tape, $20 \mathrm{~mm}$ width, located in the outer face of the glass plates. The two plates are glued together with a nylon line along the perimeter in between the plates, $2 \mathrm{~mm}$ apart of the edges. Few more nylon lines cross the inner surface as gap spacers, and also to guide the gas, see figure 4. All gluing contacts are made with epoxi resin components to obtain the right sealing of the gap formed, as well as the structural strength of the set. This construction is a solid and robust self-supported RPC, which constitutes a RPC layer of the detector modules. 

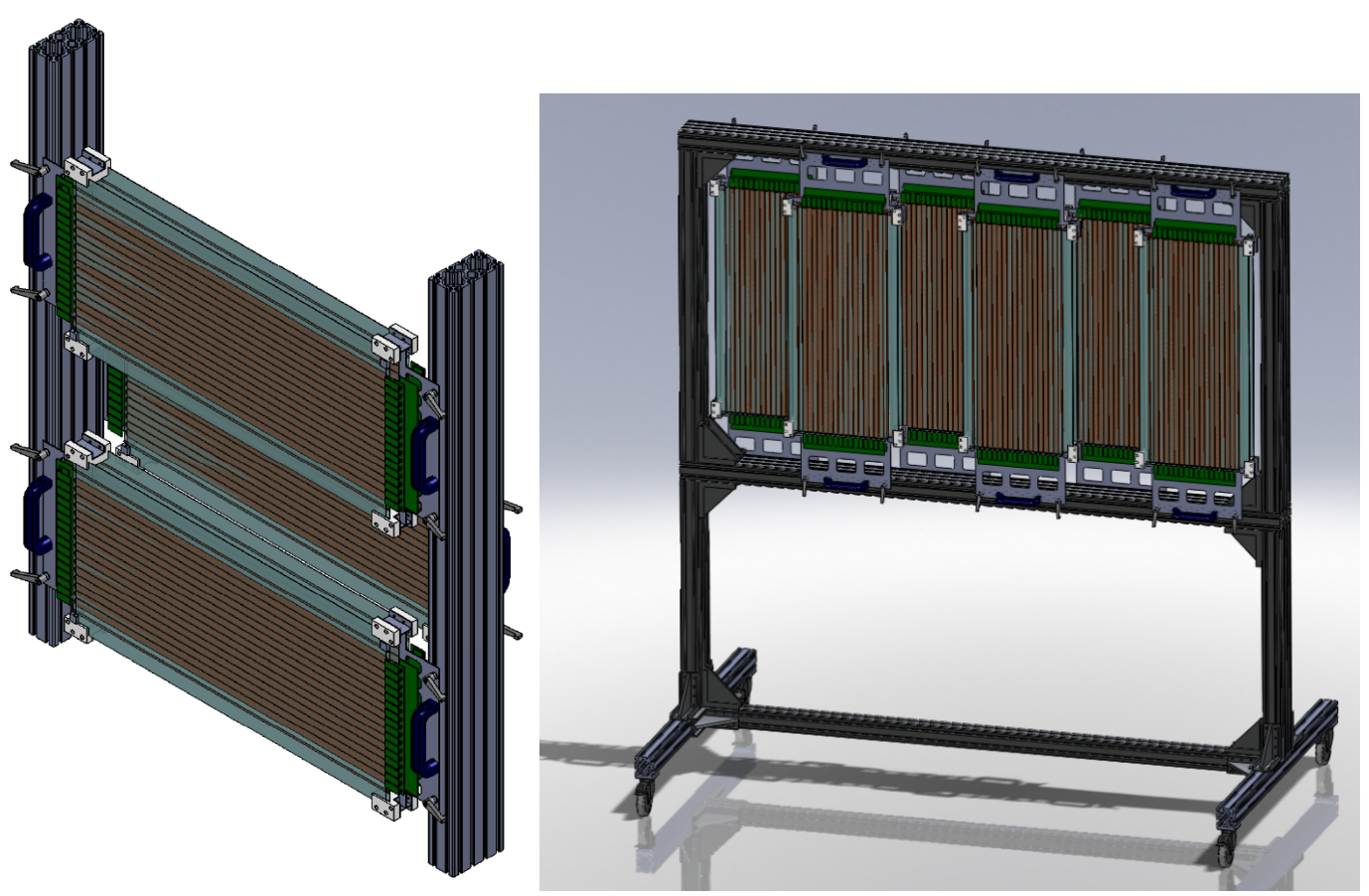

Figure 3: Design of the detector. Left panel: The modules can be safely handled and correctly positioned with simple aluminum commercial frames. A zig-zag location of next modules allows for the right overlapping to avoid dead regions. Right panel: A collection of modules can built a defined geometry and surface, with two planes of measurement. A standard configuration of six modules covers a $1 \mathrm{~m} \times 2 \mathrm{~m}$ surface to study fission residues. If more planes of measurement are needed, a second plane of detectors is used.

The strips act as electrodes and signal transmission lines. Two more strips at each side are guard strips. One of the plates is $5 \mathrm{~cm}$ longer than the other (see figure 4, right panel) allowing for an effective strip separation, for both HV (fed through a load resistor in one side, while the opposite strip is grounded) and signal pick-up at the ends. At each short side, a $5 \mathrm{~mm}$ opening is let epoxi-free so the gap is accessible. At those points we set the gas ports with a custom piece designed to serve as gas inlet in one side, and with a standard pipe connection in the other side, see figure 4, right panel. With this configuration we also make that the gas volume of the detector corresponds to its active volume $\left(120 \mathrm{~cm}^{3}\right.$ per self-supported RPC).

To achieve the granularity and position definition required, we use strips of $20 \mathrm{~mm}$ width, 22 $\mathrm{mm}$ pitch. A measurement of time side-to-side of the strip gives the longitudinal impact point. The transverse coordinate is defined by the strip position and width. The spatial resolution expected, according to the time-resolution, is enough for the purpose of the correction of the flight-path. With this geometry a RPC layer would have a $8.5 \%$ of dead space, and in the case of studying fission, up to $16 \%$ of events would hit the same strip, therefore missing the right multi-hit counting. It is the paired RPC layers concept we explained in the previous section, that overcomes those limitations, reducing the dead area to almost zero, and the coincident multi-hit to few $\%$ values. 


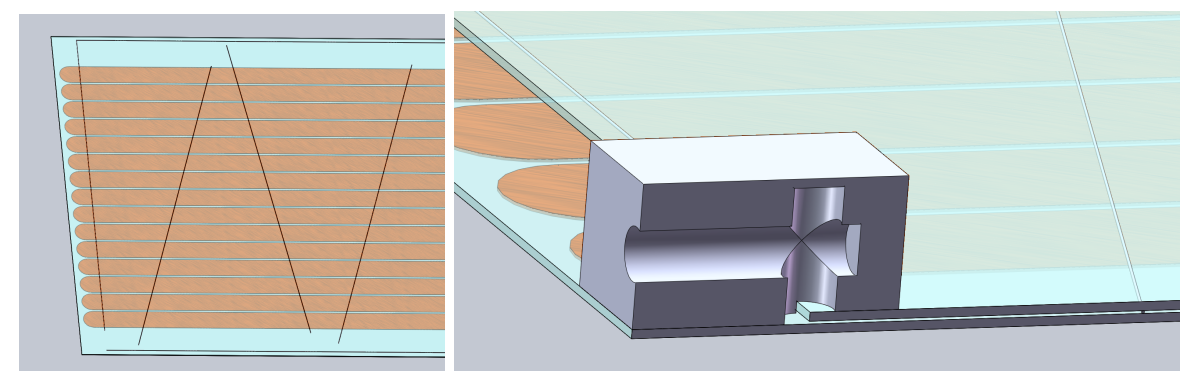

Figure 4: Design of the iTOF self-supported RPC . Left panel: A glass plate with the glued strips in the outer (lower) surface, and the nylon lines in the inner (upper) surface. The lines along the perimeter allow for sealing the gas gap with the second glass plate (not in the drawing). Other lines crossing the plate are gap spacers, and also form a gas distribution path. Right panel: Detail of a self-supported RPC, with a cross cut view (the strips of the upper plate are not shown to make a clearer drawing). A small line-free opening is let in each corner to inject the gas using a custom piece with an standard pipe size on the other side.

\section{Summary}

We proposed a feasible design for building iTOF, a detector based on RPCs to measure relativistic light and heavy ions, and suited to the activities of the R3B-FAIR experiment and other ongoing experiments at GSI. The requirements of the detector were defined with reference case studies using realistic event generators and transport simulations. We developed an R\&D program of construction and test of prototypes to study the RPC design and materials for our application.

We have designed a full modular detector, with equal units of strip RPCs. We developed the self-supported RPC concept and used structural concepts which allow for building the modules in a robust way, and with a minimal amount of matter in the active volume. The easy and safely to handle modules will be setup to cover different geometries and surfaces according to the specific kinematics of each experiment, by using appropriate frames. The detector will have several independent measuring planes to fulfill the time resolution demands with the combined resolution provided by the RPCs.

After building prototypes to test the main structural ideas as part of the R\&D, we are mounting a 1:1 scale operative module for testing in an experiment at GSI. The result will be the last step before producing the Technical Design Report of iTOF and to start the construction phase.

\section{ACKNOWLEDGEMENTS}

This work has been funded by the Ministry of Science and Innovation, Spain (project FPA200762652, and FPA2010-22174).

\section{References}

[1] A. Akindinov et al., Nuc. Inst. and Meth. A 533 (2004), 74-78.

[2] A. Schuttauf, Nuc. Ins. and Meth. A 533 (2004), 65-68.

[3] A. Blanco et al., Nuc. Inst. and Meth. A 602 (2009), 691-695.

[4] W. Yi et al., Nuc. Inst. and Meth. A 538 (2005), 425-430.

[5] P. Fonte et al., IEEE Trans. Nucl. Sci. 49 (2002), 881. 
[6] E. C. Zeballos et al., Nuc. Inst. and Meth. A 374 (1996), 132-135.

[7] P. Fonte at al., Nucl. Instr. and Meth. A 443 (2000) 201-204.

[8] H. Alvarez et al., Nuc. Phys. B Proc. Supp. 158 (2006), 186-189.

[9] Facility for Anti-proton and Ion Research, Germany. http : //www.gsi.de/fair

[10] P. Cabanelas et al., Jour. of Instr. 4 (2009) P11007.

[11] E. Casarejos et al., Nuc. Inst. and Meth. A 674 (2012), 39-45.

[12] E. Casarejos et al., Nuc. Inst. and Meth. A 661(2012) 137-140.

[13] Y. Ayyad et al., Nuc. Inst. and Meth. A 661 (2012) 141-144.

[14] C. Paradela et al., Performance of t-RPC prototypes with relativistic heavy ions, these proceedings.

[15] A review can be found in the INDC(NDS)-0539 report of the IAEA, p. 181, (2008). 